\title{
CONFORMALITY AND ISOMETRY OF RIEMANNIAN MANIFOLDS TO SPHERES
}

\author{
BY CHUAN-CHIH HSIUNG ${ }^{1}$ AND LOUIS W. STERN
}

Communicated by H. S. M. Coxeter, May 21, 1970

Let $M^{n}$ be a Riemannian manifold of dimension $n \geqq 2$ and class $C^{3}$, $\left(g_{i j}\right)$ the symmetric matrix of the positive definite metric of $M^{n}$, and $\left(g^{i j}\right)$ the inverse matrix of $\left(g_{i j}\right)$, and denote by $\nabla_{i}, R_{h i j k}, R_{i j}=R_{i j k}^{k}$ and $R=g^{i j} R_{i j}$ the operator of covariant differentiation with respect to $g_{i j}$, the Riemann tensor, the Ricci tensor and the scalar curvature of $M^{n}$ respectively. Throughout this paper all Latin indices take the values $1, \cdots, n$ unless stated otherwise. We shall follow the usual tensor convention that indices can be raised and lowered by using $g^{i j}$ and $g_{i j}$ respectively, and that repeated indices imply summation.

Let $v$ be a vector field defining an infinitesimal conformal transformation on $M^{n}$. Denote by the same symbol $v$ the 1 -form corresponding to the vector field $v$ by the duality defined by the metric of $M^{n}$, and by $L_{v}$ the operator of the infinitesimal transformation $v$. Then we have

$$
L_{v} g_{i j}=\nabla_{i} v_{j}+\nabla_{j} v_{i}=2 \rho g_{i j} .
$$

The infinitesimal transformation $v$ is said to be homothetic or an infinitesimal isometry according as the scalar function $\rho$ is constant or zero. We also denote by $L_{d \rho}$ the operator of the infinitesimal transformation generated by the vector field $\rho^{i}$ defined by

$$
\rho^{i}=g^{i j} \rho_{j}, \quad \rho_{j}=\nabla_{j} \rho .
$$

Let $\xi_{i_{1}} \ldots i_{p}$ and $\eta_{i_{1}} \ldots i_{p}$ be two tensor fields of the same order $p \leqq n$ on a compact orientable manifold $M^{n}$. Then the local and global scalar products $\langle\xi, \eta\rangle$ and $(\xi, \eta)$ of the tensor fields $\xi$ and $\eta$ are defined by

$$
\langle\xi, \eta\rangle=\frac{1}{p !} \xi^{i_{1} \cdots i_{p}} \eta_{i_{1} \cdots i_{p}},
$$

AMS 1970 subject classifications. Primary 5325, 5372; Secondary 5747.

Key words and phrases. Infinitesimal nonisometric conformal transformations, scalar curvature, lengths of Riemann and Ricci curvature tensors.

${ }^{1}$ Work partially supported by NSF grant GP-11965. 


$$
(\xi, \eta)=\int_{M^{n}}\langle\xi, \eta\rangle d V
$$

where $d V$ is the element of volume of the manifold $M^{n}$ at a point.

In the last decade or so various authors have studied the conditions for a Riemannian manifold $M^{n}$ of dimension $n>2$ with constant scalar curvature $R$ to be either conformal or isometric to an $n$-sphere. Very recently Yano, Obata, Hsiung and Mugridge (see [6], [4], [2]) have been able to extend some of the above-mentioned results by replacing the constancy of $R$ by $L_{u} R=0$, where $u$ is a certain vector field on $M^{n}$. The purpose of this paper is to continue their work by establishing the following theorems.

To begin we denote by $(C)$ the following condition:

A compact Riemannian manifold $M^{n}$ of dimension $n>2$ (C) admits an infinitesimal nonisometric conformal transformation $v$ satisfying (1.1) with $\rho \neq 0$ such that $L_{v} R=0$.

Theorem I. An orientable $M^{n}$ is conformal to an $n$-sphere if it satisfies condition $(\mathrm{C})$ and

$$
\begin{aligned}
& \left(\rho_{i} \rho^{i}-\frac{1}{n-1} R \rho^{2}, R\right) \geqq 0, \\
& L_{v}\left(a^{2} A+\frac{c-4 a^{2}}{n-2} B\right)=0,
\end{aligned}
$$

where $A$ and $B$ are defined by

$$
A=R^{h i j k} R_{h i j k}, \quad B=R^{i j} R_{i j},
$$

and $a, c$ are constant such that

$$
\begin{aligned}
\begin{array}{l}
c \equiv 4 a^{2}+(n-2) \\
(1.8)
\end{array} & {\left[2 a \sum_{i=1}^{4} b_{i}+\left(\sum_{i=1}^{6}(-1)^{i-1} b_{i}\right)^{2}\right.} \\
& \left.-2\left(b_{1} b_{3}+b_{2} b_{4}-b_{5} b_{6}\right)+(n-1) \sum_{i=1}^{6} b_{i}^{2}\right]>0,
\end{aligned}
$$

$b$ 's being any constants.

For the case $a \neq 0, c-4 a^{2}=0$ and the case $a=0, c-4 a^{2} \neq 0$, Theorem $I$ is due to Yano [4].

Theorem II. A manifold $M^{n}$ is conformal to an $n$-sphere, if it

${ }^{2}$ An elementary calculation shows that $c \geqq 0$ where equality holds if and only if $b_{1}=\cdots=b_{4}, b_{5}=b_{6}=0, a=-(n-2) b_{1}$. 
satisfies condition (C) and any one of the following three sets of conditions:

$$
\begin{aligned}
\nabla_{i} \nabla_{j}(R f) & =R \rho g_{i j} & & (f \text { is a scalar function }), \\
Q d \rho & =\frac{2}{n} d(R \rho), & & \nabla_{i} \nabla_{j}(R \rho)=R \nabla_{i} \nabla_{j} \rho, \\
L_{v} R_{i j} & =\alpha g_{i j} & & (\alpha \text { is a scalar function }),
\end{aligned}
$$

where $Q$ is the operator of Ricci defined by, for any vector field $u$ on $M^{n}$,

$$
Q: u_{i} \rightarrow 2 R_{i j} u^{j} \text {. }
$$

For constant $R$, conditions (1.10) and (1.11) in Theorem II will lead to the conclusion that $M^{n}$ is isometric to an $n$-sphere of radius $(n(n-1) / R)^{1 / 2} ;$ for this see $[5]$.

Theorem III. A manifold $M^{n}$ with constant $R$ is isometric to an $n$ sphere of radius $(n(n-1) / R)^{1 / 2}$, if it satisfies conditions (C) and (1.9).

Theorem III is due to Lichnerowicz [3] when condition (1.9) is replaced by the following one:

(1.13) $v$ is the gradient of a scalar function $f$, i.e., $v_{i}=\nabla_{i} f$.

For constant $R$, it is easily seen that condition (1.13) is a special case of condition (1.9). In fact, in this case by using (1.2) condition (1.9) becomes $\nabla_{i} v_{j}+\nabla_{j} v_{i}=2 \nabla_{i} \nabla_{j} f$, which is satisfied by $v_{i}=\nabla_{i} f+u_{i}$ where $u_{i}$ is any vector field generating an infinitesimal isometry.

Theorem IV. A manifold $M^{n}$ is isometric to an $n$-sphere, if it satisfies condition $(\mathrm{C}), L_{d \rho} R=0$, and

$$
\begin{aligned}
A^{a} B^{b}= & c=\text { const, } \\
c\left(\frac{2 a}{A}+\frac{(n-1) b}{B}\right) & =\frac{2^{a}(a+b) R^{2(a+b-1)}}{n^{a+b-1}(n-1)^{a-1}},
\end{aligned}
$$

where $A, B$ are given by (1.7), and $a, b$ are nonnegative integers and not both zero.

For constant $R$, Theorem IV is due to Lichnerowicz [3] for $a=0$, $b=1$ and due to Hsiung [1] for general $a$ and $b$.

\section{BibLIOGRAPHY}

1. C.-C. Hsiung, On the group of conformal transformations of a compact Riemannian manifold, Proc. Nat. Acad. Sci. U.S.A. 54 (1965), 1509-1513. MR 32 \#6372.

2. C.-C. Hsiung and L. R. Mugridge, Conformal changes of metrics on a Riemannian manifold, Math. Z.(to appear). 
3. A. Lichnerowicz, Sur les transformations conformes d'une variete riemannienne compacte, C. R. Acad. Sci. Paris 259 (1964), 697-700. MR 29 \#4007.

4. K. Yano, On Riemannian manifolds admitting an infinitesimal conformal transformation, Math. Z. 113 (1970), 205-214.

5. K. Yano and M. Obata, Sur le groupe de transformations conformes d'une variété de Riemann dont le scalaire de courbure est constant, C. R. Acad. Sci. Paris 260 (1965), 2698-2700. MR 31 \#697.

6. - Conformal changes of Riemannian metrics, J. Differential Geometry 4 (1970), 53-72.

Lehigh University, Bethlehem, Pennsylvania 18015 